\title{
Local Electrode Atom Probe Tomography of Carbon Fibre
}

\author{
R.K.W. Marceau ${ }^{1 *}$, A.S. Taylor ${ }^{1}$, T. Sato ${ }^{2}$, S.P. Ringer ${ }^{2}$, B.L. Fox ${ }^{3}$, N. Stanford ${ }^{4}$ and L.C. Henderson ${ }^{1}$ \\ 1. Deakin University, Institute for Frontier Materials, Geelong, VIC 3216, Australia. \\ 2. Australian Centre for Microscopy and Microanalysis, University of Sydney, NSW, 2006, Australia. \\ 3. Swinburne University of Technology, Hawthorn, VIC 3122, Australia. \\ 4. Future Industries Institute, University of South Australia, Mawson Lakes, SA 5095, Australia. \\ * Corresponding author: ross.marceau@deakin.edu.au
}

Carbon fibre composites are increasingly being utilised across a wide range of industries. Currently there is a gap in knowledge concerning the micro- and nano-scale structure-property relationships that govern the mechanical properties of the individual carbon fibres and therefore also the behaviour of the composite material. Atom probe tomography (APT) is uniquely placed to address this situation because it provides a combination of highly resolved chemical and spatial information in three dimensions with the ability to detect all the elements (and their isotopes) in the periodic table. APT is thus a very suitable technique for analysis of carbon fibres, which are mostly comprised of light elements (namely $\mathrm{C}, \mathrm{H}, \mathrm{N}$, and $\mathrm{O}$ ) that are otherwise very challenging to detect in combination with each other using other microscopy and microanalysis techniques, pushing the limits of detection with respect to spatial resolution, chemical sensitivity and mass resolving power.

In the current work, the structure of TORAYCA-M46J polyacrylonitrile (PAN)-based fibre (individual fibre diameter $7 \mu \mathrm{m}$ ) has been examined using APT. After isolating an individual fibre, specimen preparation for APT was carried out using a combination of focused ion beam (FIB) and scanning electron microscopy (SEM) to produce the required sharp tip with an end radius of less than $50 \mathrm{~nm}$. Atom probe data acquisition was carried out in both voltage and laser pulse mode using local electrode atom probe (LEAP) instruments at Deakin (LEAP 4000 HR and recently upgraded to LEAP 5000 XR) and Sydney (LEAP 4000X Si) Universities.

Mass spectra after laser pulsing were much better resolved than those acquired using voltage pulsing, with a reduced incidence of fracture. There is, however, a significant challenge for accurate and unambiguous mass spectral analysis given the rate of incidence of detection of carbon molecular ions and complex molecular ionic species (Fig. 1), which often occur on multiple hit events. Through novel peak assignment analysis, the current work demonstrates that laser-pulsed atom probe tomography provides atom-sensitive composition assessment of carbon fibre, accurate to within $~ 5.3 \mathrm{wt} . \%$.

The potentials used in the APT technique are typically at the $\mathrm{kV}$ level, which is more than adequate to cause substantial fragmentation of the molecular species of which this material is composed. The complexity of field evaporation in this case is highlighted by the histogram of correlated field evaporated ions [1] shown in Fig. 2, where a significant number of curved tracks are observed and these are signatures of molecular ion dissociation. While field-induced molecular fragmentation may be viewed as a drawback, analysis of these products facilitates a basis for comparison of key fragments (such as $\mathrm{C}=\mathrm{O}$ ) between different fibres or fibre treatments, and also for comparison of this type of APT data with that from other characterisation techniques typically used to investigate carbon fibre, such as X-ray photoelectron spectroscopy (XPS) [2,3] and time-of-flight secondary ion mass spectroscopy (TOF-SIMS) [3,4]. 


\section{References:}

[1] DW Saxey, Ultramicroscopy 111 (2011), p. 473.

[2] MR Alexander and FR Jones, Carbon 32 (1994), p. 785.

[3] E Desimoni et al., J Electron Spectrosc 85 (1997), p. 179.

[4] MR Alexander and FR Jones, Carbon 34 (1996), p. 1093.

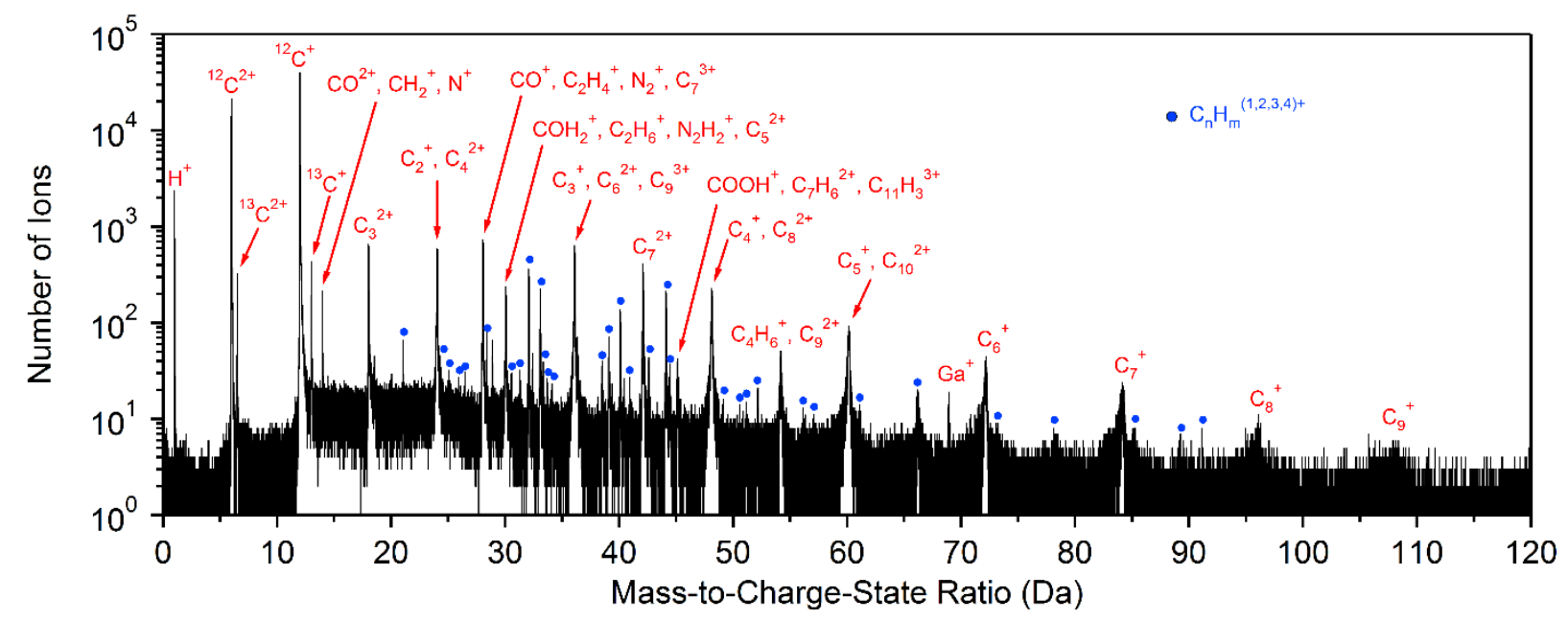

Figure 1. LEAP 4000X Si mass spectrum of carbon fibre collected in laser-pulsed mode.
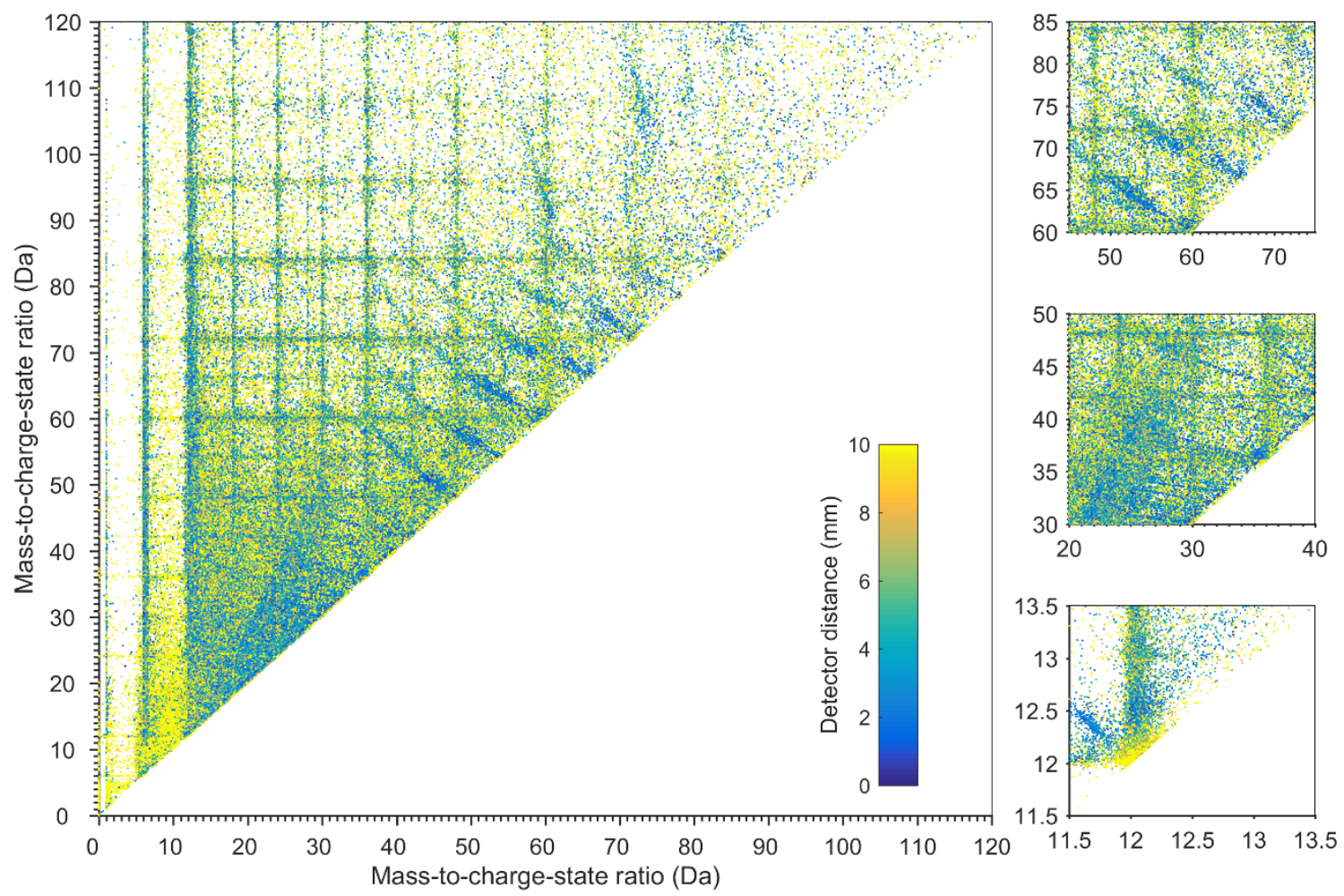

Figure 2. Ion correlation histogram from all pairs of ions within multiple hit events in a carbon fibre dataset collected using a LEAP 4000X Si instrument. 